\title{
INTERACTION OF ELECTROMAGNETIC AND ACOUSTIC WAVES IN CONDUCTORS AND ITS UTILIZATION
}

The paper deals with the interaction of electromagnetic and acoustic processes in a conducting medium in presence of an external magnetic field. The electromagnetic excitation of acoustic wave similarly as acoustic excitation of electromagnetic wave is described. The practical utilization consisting in contactless acoustic wave generation and detection is demonstrated.

\section{Introduction}

The elastic and conducting medium consists of ionic lattice and gas of electrons. The external electromagnetic field acts on the particles and excites their motion. Under usual conditions the direct electromagnetic force affecting the ionic structure is screened by the electron collisions drag force, so that no mechanical excitation is observed. The penetrating electromagnetic wave is strongly damped in a thin surface skin layer. The screening effect can be disturbed by an external constant magnetic field. In such a case the mechanical excitation of the ionic lattice can propagate through the sample as an elastic wave, e.g. [1]. On the other hand the ionic movement generates the secondary electromagnetic wave which accompanies the elastic wave and represents the elastic mode of the electromagnetic wave, e.g. [2]. It propagates with the acoustic wave velocity and weak attenuation and can be detected at the opposite side of the conducting sample. There are many possibilities of the utilization of the effect. One of them consists in contactless ultrasonic defectoscopy of metal samples, another in the electromagnetic detection of mechanical vibrations in bodies, etc. The effect can be utilized in a wide range of frequencies and temperatures.

\section{Theoretical description of the effect}

Interaction of the mechanical motion of the structure and electromagnetic field in the medium can be described by means of wave equations of the electromagnetic wave and the elastic wave

$$
\begin{aligned}
& \Delta E-\epsilon \mu \frac{\partial^{2} E}{\partial t^{2}}=\mu \frac{\partial J}{\partial t} \text { and } \\
& c_{l} \operatorname{grad} \operatorname{div} \xi-c_{t} \operatorname{rot} \operatorname{rot} \xi-\rho \frac{\partial^{2} \xi}{\partial t^{2}}=f,
\end{aligned}
$$

where $\boldsymbol{E} \equiv \boldsymbol{E}(\boldsymbol{r}, t)$ is the electric intensity, $\xi \equiv \xi(\boldsymbol{r}, t)$ is the ionic displacement from the equilibrium state, $\epsilon$ and $\mu$ are permitivity and permeability of the medium, $c_{l}$ and $c_{t}$ are longitudinal and shear elastic constants, $\rho$ is the density and $f$ is the density of non- elastic forces acting on ions. $\boldsymbol{J} \equiv \boldsymbol{J}(\boldsymbol{r}, t)$ is the electric current density, which consists of both ionic and electronic parts

$$
\boldsymbol{J}(\boldsymbol{r}, t)=\boldsymbol{J}_{i}(\boldsymbol{r}, t)+\boldsymbol{J}_{\boldsymbol{e}}(\boldsymbol{r}, t) .
$$

Ionic current is in connection with ionic displacement velocity

$$
\boldsymbol{J}_{i}(\boldsymbol{r}, t)=n e \frac{\partial \xi}{\partial t} .
$$

Electronic current density is given by

$$
J_{e}(\boldsymbol{r}, t)=\vec{\sigma} \cdot\left(\boldsymbol{E}-\frac{n e}{\sigma_{0}} \frac{\partial \xi}{\partial t}+\frac{2 E_{F}}{3 e} \operatorname{grad} \operatorname{div} \xi\right),
$$

where $\vec{\sigma}$ is the tensor of the magneto-conductivity, $\sigma_{0}$ is the DC conductivity, $n$ and $e$ are the electron density and the elementary charge and $E_{F}$ is the Fermi energy of the electron gas. The second term in the parentheses corresponds to the electron current caused by the motion of the ionic background and the third to the diffusion of the electrons.

The resulting force density acting on the ionic lattice can be expressed as

$$
\boldsymbol{f}(\boldsymbol{r}, t)=f_{E M}(\boldsymbol{r}, t)+f_{\boldsymbol{e}}(\boldsymbol{r}, t),
$$

where

$$
f_{E M}(\boldsymbol{r}, t)=n e\left(\boldsymbol{E}+\frac{\partial \xi}{\partial t} \times \boldsymbol{B}\right)
$$

is electromagnetic Lorentz force density from the resulting electromagnetic field in the medium and

$$
f_{e}(\boldsymbol{r}, t)=-\frac{n m}{\tau}\left(\frac{1}{n e} \boldsymbol{J}_{e}+\frac{\partial \xi}{\partial t}\right)
$$

is the electron drag force resulting from the electron-ion collisions. In two last terms there is $\boldsymbol{B}$ the magnetic flux density, $m$ is the mass of electron and $\tau$ is the collision mean free time.

The dynamic process can be significantly influenced by means of external constant magnetic field. In the case of linear processes

\footnotetext{
* ${ }^{1}$ prof. Ing. Ivo Čáp, CSc., ${ }^{2}$ prof. Ing. Klára Čápová, PhD., ${ }^{2}$ Ing. Dagmar Faktorová

${ }^{1}$ University of Žilina, Faculty of Science, Hurbanova 15, SK-010 26 Žilina, Slovakia, E-mail: icap@fpv.utc.sk

${ }^{2}$ University of Žilina, Faculty of Electrical Engineering, Vel'ký diel, SK-010 26 Žilina, Slovakia, E-mail: capova@fel.utc.sk
} 
the magnetic flux density $\boldsymbol{B}$ will be substituted by the constant value $\boldsymbol{B}_{c}$ and the time-dependent component will be neglected. In the presence of the external magnetic filed the conductivity tensor represents the electric anisotropy of the medium depending on the magnetic field orientation. For example, if the magnetic field vector $\boldsymbol{B}_{c}$ is parallel to the $\mathrm{z}$ direction, the magneto-conductivity tensor has the form

$$
\vec{\sigma}=\sigma_{0}\left(\begin{array}{ccc}
1 & 0 & 0 \\
0 & 1 & -\omega_{c} \tau \\
0 & \omega_{c} \tau & 1
\end{array}\right),
$$

where $\omega_{c}=e B_{c} / m$ is the cyclotron angular frequency.

The products of the conductivity tensor $\vec{\sigma}$ and the terms obtaining $\xi$ in (5) together with vector product in (7) represent the mathematical description of the mechanism of the interaction of electromagnetic and mechanic processes in the medium. In such way the time-dependent electromagnetic excitation generates acoustic process in the ionic structure and on the other hand the mechanical motion of the ionic structure leads to the accompanying electromagnetic field generation.

\section{Waves in a conducting medium}

Let us suppose the semi-infinite sample with the surface normal to the $z$-direction and all wave processes as plane waves propagating in $z$-direction. The set of equations (1) and (2) together with (3) to (8) can be solved under corresponding boundary conditions by means of Laplace transformation, e.g. [2], [3]

In many practical applications we utilise the mechanism of electromagnetic generation of the acoustic wave. In such case we suppose the plane electromagnetic wave polarized in $x$-direction and incident perpendicularly to the sample surface. In the case of $\boldsymbol{B}_{c}$ parallel to the $z$-direction the shear acoustic wave with $y$-polarization is excited. If the $\boldsymbol{B}_{c}$ has y-direction, the longitudinally polarized acoustic wave is excited. The time and space dependence can be expressed by

$$
\xi(z, t)=i \frac{2 H_{0} B_{c}}{\omega \rho s} e^{-i \frac{\omega}{s} z} e^{i \omega t},
$$

where $H_{0}$ is the magnetic intensity of the incident electromagnetic wave at the surface, $\omega$ is the angular frequency and $s$ is the veloc ity of the acoustic wave of the corresponding polarization. The shear component of the accompanying electromagnetic wave perpendicular to the propagation direction $\mathrm{z}$ can be expressed by

$$
E(z, t)=i \omega B_{c} \xi(z, t)=i \omega B_{c} \xi_{0} e^{-i \frac{\omega}{s} z} e^{i \omega t},
$$

where $\xi_{0}$ is the acoustic wave amplitude at the surface of the sample

The electromagnetic wave accompanying the electromagnetically excited acoustic wave is given by

$$
E(z, t)=\frac{2 H_{0} B_{c}^{2}}{\rho s} e^{-i \frac{\omega}{s} z} e^{i \omega t} .
$$

In the case of planparallel sample the electromagnetic wave is radiated from the sample and it can be detected by a proper detecting coil.

These results are exactly valid for non-magnetic materials. In the case of ferromagnetic materials these processes are influenced by magnetisation and magnetostriction.

\section{Electromagnetic - acoustic transducer}

The described effect is used in many practical applications. One of them consists in one-probe electromagnetic generation and detection of ultrasound. The arrangement of the probe called electromagnetic-acoustic transducer (EMAT), e.g. [4], is in Fig. 1.
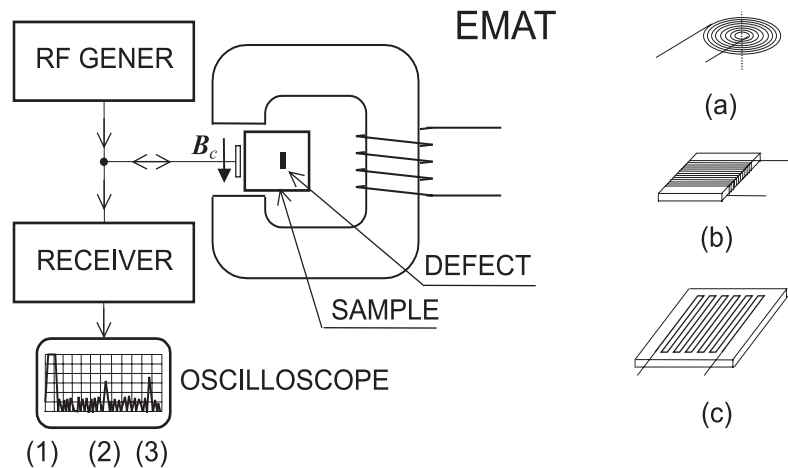

(b)

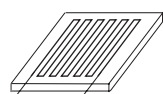

(c)

(1) (2) (3)

Fig. 1. Arrangement of EMAT and the shape of generating/detecting coils

The EMAT is mostly used for the ultrasonic defectoscopy. RF pulse generates the starting ultrasonic pulse (1). It propagates across the sample and reflects from the backside of the sample, see pulse (3) or from the defects of the sample (2). All pulses are detected, processed by a receiver and visualised by means of an oscilloscope. Position of the defect inside the sample is measured by means of time-delay of the reflected pulse. The conducting sample is placed in the magnetic field of a permanent magnet or an electromagnet. The sample can be placed into the magnetic field so that the magnetic flux density is normal or parallel to the generating-detecting surface of the sample. It determines the slower shear or the faster longitudinal acoustic wave generation.

Both acoustic and accompanying electromagnetic waves were generated by proper coils, Fig. 1 (a) to (c), supplied by the RF signal generator. The coil design determines the acoustic beam profile. The most complex beam structure but the best efficiency represents the flat spiral coil, Fig. 1 (a) and Fig. 2. In the case of a constant magnetic field parallel to the surface, Fig. 2 (a) the generated beam consists of two sub-beams with opposite longitudinal polarization. If $\boldsymbol{B}_{c}$ is normal to the surface, the generated beam has a shear radial polarization with zero value along the axis of the acoustic beam. This property can be utilised in special applications of EMAT. 


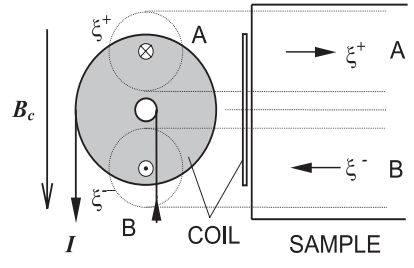

(a)

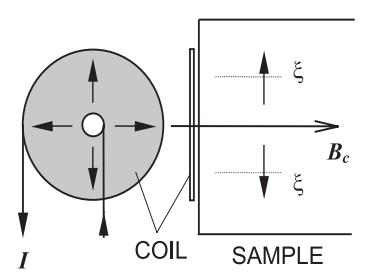

(b)
Fig. 2. Polarization of the acoustic wave generated by the flat spiral coil

Another typical shape of the coil is in Fig. 3. The generating/detecting coil is made from the thin wire wound in one layer around the flat dielectric strip. The RF magnetic fields of both layers of the winding with opposite directions of the current and with the phase shift $k d$ mutually interfere. The resulting RF magnetic field at the surface of the conducting sample is reduced by $k d=\omega d / c$ in comparison with the one-layer coil, e.g. the mentioned spiral coil. The advantage of the rectangular coil is the generation of very homogeneous acoustic beam similar to the plane wave with a better defined wave polarization in the whole sample. The sensitivity is lower than in the case of the spiral coil. Due to the reduction factor $k d$ this generating/detecting coil can be used for higher frequencies applications. The system was tested in the frequency range from 10 to $25 \mathrm{MHz}$.
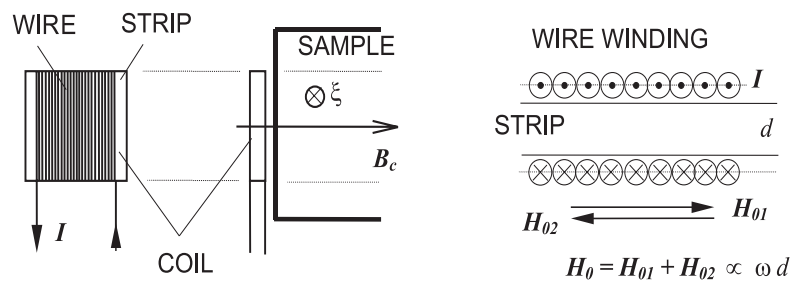

Fig. 3. Rectangular coil

The system of a flat wire coil allows to make generating/detecting coils for the special acoustic waves, as deflected waves, surface waves, focused waves, etc. Fig. 4. The coil in Fig. 4 (a) is able to generate or to detect the acoustic wave deflected from the direction normal to the surface by angle $\theta$ according to the ratio of the acoustic wave-length and the period $x$ of the coil structure. The surface acoustic wave is generated under condition of $x=\lambda$. The structure in Fig. 4 (b) generates or detects the focused cylindrical wave. Such a system is very sensitive for the detection of material defects in the corresponding depth. The similar coil can be made as a circular one and then it serves as a planar acoustic lens used in the acoustic microscopy. Special coils can be made by means of planar technology in one-layer or as wire-coils. Because of the exact phase conditions the mentioned coils are designed for concrete frequency. We investigated the metal samples by means of surface acoustic waves at the frequency range 9.8 and $15 \mathrm{MHz}$. The focused cylindrical system with the tunable focus-length in the range from 5 to $8 \mathrm{~mm}$ was used for the one-dimensional scanning system at the mean frequency of $10 \mathrm{MHz}$. The reached resolution was about $0.5 \mathrm{~mm}$.
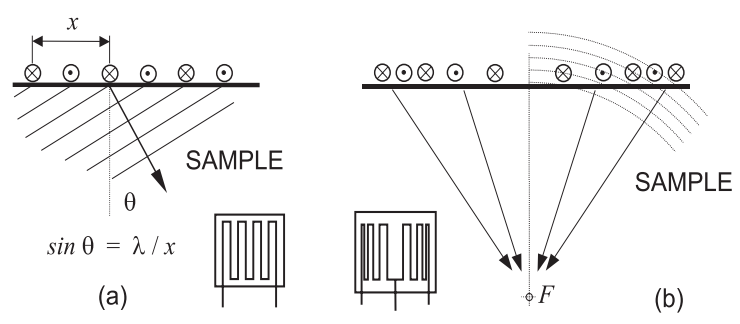

Fig. 4. Special generating/detecting coils

The experimental arrangement of the EMAT had the typical values of characteristic parameters: the RF frequency from 1 to 25 $\mathrm{MHz}$, the generating pulse length of 2 to $5 \mu \mathrm{s}$, the repetition rate of $1 \mathrm{kHz}$, the electric current pulse amplitude of about $10 \mathrm{~A}$. Because of the typical coil resistance of about $5 \Omega$ it represents the pulse power of about $500 \mathrm{~W}$. The received signal emitted from the sample was detected with the same coil and it was amplified by the wide band amplifier with the amplification of $60 \mathrm{~dB}$.

The signal efficiency increases with decrease of the frequency mainly due to the decrease of the elastic wave attenuation, but the resolution increases with the increase of the frequency because of decrease of the acoustic wavelength.

\section{Conclusions}

The system of EMAT is suitable for investigation of electromagnetic and electrodynamic properties of different conducting structures. The obtained results were utilized in the construction of the EMAT, which enables to investigate defects of conducting samples. The defectoscopy using the EMAT makes possible to investigate the structure of the sample without any acoustic coupling, which is advantageous for the scanning method of the sample structure visualization. The coils made on the different substrates are useable in a wide temperature range as well. Contactless defectoscopy is proper for the investigation of different bodies in motion, e.g. rails or railway wheels. Other applications are connected with special shapes of acoustic beam generated by special generating coils. 


\section{References}

[1] ARONOV, I. E., FALKO, V. L.: Electromagnetic Generation of Sound in Metals in a Magnetic Field. Physics Reports 221 (1992), No. 2-3, pp. $81-166$.

[2] ČÁPOVÁ, K., ČÁP, I.: Acoustically excited EM wave in metal, in: Studies in Applied Electromagnetics and Mechanics, vol. 13, V. Kose and J. Sievert, eds. IOS Press, Amsterdam, 1998, pp. 811-814.

[3] ČÁPOVÁ, K., ČÁP, I.: Material anisotropy investigation using anomalous EM generation of acoustic wave, in: Studies in Applied Electromagnetics and Mechanics, vol. 14, R. Albanese at all, eds. IOS Press, Amsterdam, 1998, pp. 74-81.

[4] OGI, H.: Field dependence of coupling efficiency between electromagnetic field and ultrasonic bulk waves. J. Appl. Phys. 82, No 8 (1997), 3940-3949. 